\title{
Oxycodone-Related Deaths: The Significance of Pharmacokinetic and Pharmacodynamic Drug Interactions
}

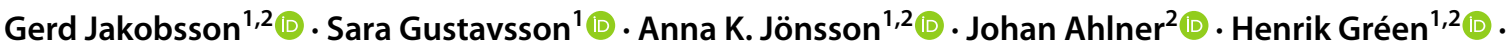 \\ Robert Kronstrand ${ }^{1,2}$ (ID
}

Accepted: 15 December 2021 / Published online: 13 January 2022

(c) The Author(s) 2022

\begin{abstract}
Background and Objectives Oxycodone is frequently prescribed as well as detected in postmortem cases. Concurrent use of pharmacodynamically or pharmacokinetically interacting drugs can cause adverse effects or even fatal intoxication. The aims of this study were to investigate differences in prescriptions for and toxicological findings of pharmacodynamically and pharmacokinetically interacting drugs in fatal oxycodone-related intoxications and other causes of death. We also aimed to investigate the differences in prevalence of oxycodone prescriptions, and the detected postmortem oxycodone concentrations between fatal oxycodone-related intoxications and other causes of death.

Methods Forensic autopsy cases (2012-2018) where oxycodone was identified in femoral blood $(n=1236)$ were included. Medical history and prescription data were retrieved from national databases and linked to the forensic toxicology findings. Results Oxycodone-related deaths were found to have higher blood concentrations of oxycodone (median $0.30 \mu \mathrm{g} / \mathrm{g}$ vs. $0.05 \mu \mathrm{g} / \mathrm{g}$ ) and were less likely to have a prescription for oxycodone (OR 0.62) compared to nonintoxication deaths. Pharmacodynamically interacting drugs were prescribed in $79 \%$ and found in blood in $81 \%$ of the cases. Pharmacokinetically interacting drugs were rarely prescribed (1\%). Oxycodone-related deaths were more likely to have prescriptions for a pharmacodynamically interacting drug (OR 1.7) and more often have co-findings of one or multiple pharmacodynamically interacting drugs (OR 5.6).

Conclusion The results suggest that combined use of oxycodone and pharmacodynamically interacting drugs is associated with oxycodone-related death and that non-medical use of oxycodone is a potential risk factor for oxycodone-related intoxication.
\end{abstract}

Gerd Jakobsson

gerd.jakobsson@liu.se

1 Department of Forensic Genetics and Forensic Toxicology, National Board of Forensic Medicine, 58758 Linköping, Sweden

2 Division of Clinical Chemistry and Pharmacology, Department of Biomedical and Clinical Sciences, Linköping University, 58183 Linköping, Sweden

\section{Key Points}

In oxycodone-related deaths, $41 \%$ did not have a prescription for oxycodone, suggesting misuse as a risk factor.

Drugs impairing the metabolism of oxycodone were very rarely prescribed.

Compared to other deaths, persons dying from oxycodone intoxication were 5.6 times more likely to have other CNS depressant drugs on board and 1.7 times more likely to have them prescribed. 


\section{Introduction}

\subsection{Prescription and Use}

Opioid analgesics are used world-wide in clinical practice for the treatment of acute and chronic pain. In reports from the USA, Australia, Canada, and the EU, increased prescribing and use have led to increasing numbers of opioid-related deaths [1-5]. Dispensing of prescribed opioids has also been studied in the Nordic countries [6,7]. Müller et al. studied trends in dispensing of prescribed opioids in Norway, Denmark, and Sweden between 2006 and 2017 [7]. The authors reported that oxycodone prescription increased in Norway $(0.2-0.8 \%)$ and Sweden $(0.4-3 \%)$ during the time period [7]. In a study by Bäckryd et al., it was observed that the number of patients receiving tramadol during the studied period (2000-2015) decreased 54\%, but, in contrast, a 465\% increase of patients receiving oxycodone was observed [6]. More recent data of oxycodone from the National Board of Health and Welfare are displayed in Fig. 1, showing the increase of oxycodone prescriptions dispensed, which increased from 15 patients per 1000 inhabitants in 2012 to 37 in 2018.

\subsection{Misuse and Death}

As the availability of prescription opioids has increased, so has the misuse and their associated consequences [2, 3, 8, 9]. Centers for Disease Control and Prevention reported that nearly $30 \%$ of all opioid overdose deaths in 2019 in the US involved a prescription opioid [8]. Although prescription opioids are involved in many deaths, they are not necessarily prescribed $[2,9]$. This has been shown for oxycodone by several research groups $[3,9,10]$. Pilgrim et al. reported a sevenfold increase in oxycodone-related deaths in Australia between 2001 and 2011. However, of the 806 oxycodonerelated deaths, as many as $61 \%$ of the cases did not have a legitimate prescription for oxycodone [3]. Ogle et al. studied the toxicological findings of 155 oxycodone-related deaths to determine demographic characteristics and clinical background. They found that 53\% did not hold a legitimate prescription for oxycodone [10]. Non-prescribed or illicit use of oxycodone might lead to higher risk of unwanted effects both related and unrelated to interactions with other drugs.

\subsection{Pharmacokinetic Interactions}

The extensive metabolism of oxycodone in the liver by cytochrome P450 (CYP)3A4 and CYP2D6 makes oxycodone susceptible to pharmacokinetic drug-drug interactions (PKI) [11] Kim et al. studied the oxycodone-related adverse effects and PKI associated with CYP P450 enzymes in 111 geriatric patients. They found that $32.4 \%$ experienced at least one adverse effect, and that use of either CYP2D6 inhibitors or CYP3A4 inhibitors increased the rate of adverse effects by 20.4 and 25.4 times (odds ratio), respectively, suggesting that PKIs are a clinical relevant issue [12]. In another study, drug-drug interactions (DDIs) were studied by a physiologically-based pharmacokinetic modeling. The authors suggested from
Fig. 1 Trends (2012-2018) in the number of postmortem cases positive for oxycodone in femoral blood, and the number of patients per 1000 inhabitants prescribed oxycodone, stratified by oxycodone-related intoxications (INTOX), death by other causes (NONINTOX), and intoxications unrelated to oxycodone (OTHER). Compiled data from National Board of Health and Welfare and National Board of Forensic Medicine

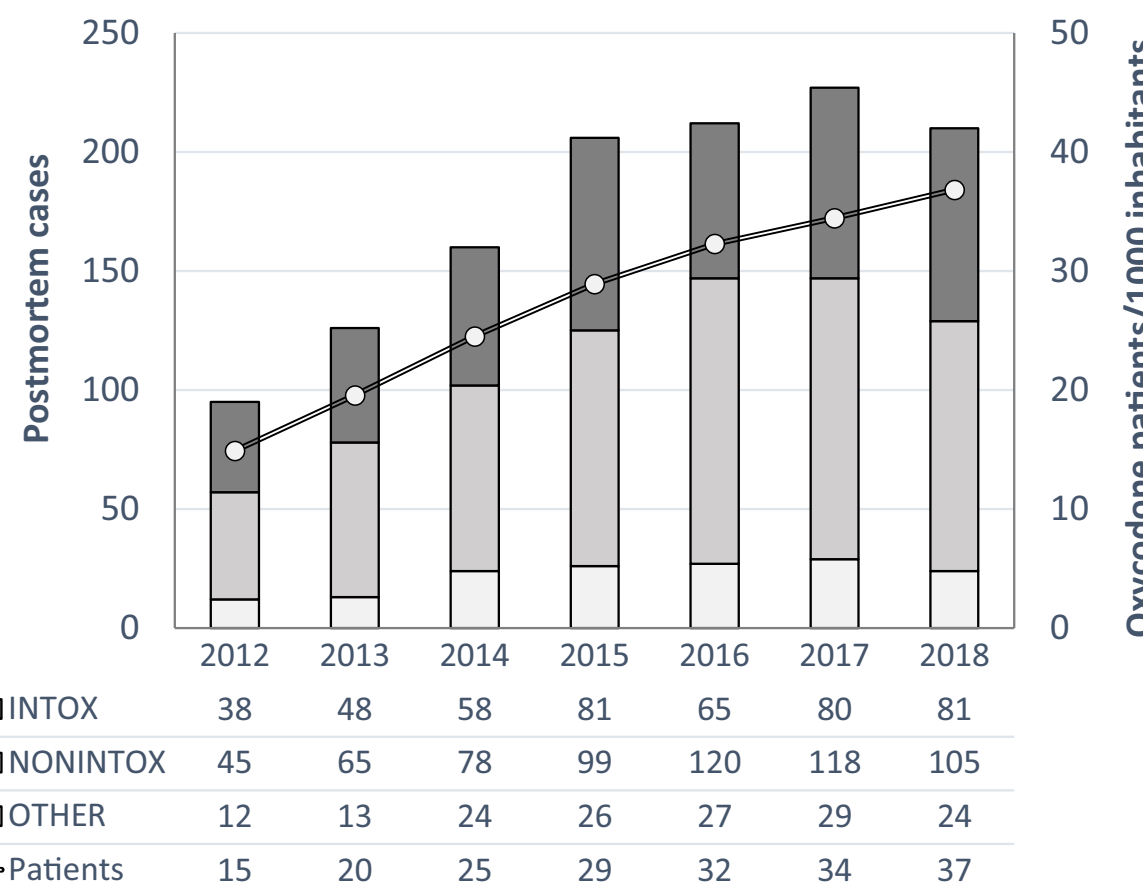


their results that pharmacodynamic drug-drug interactions (PDI) might be of greater importance than PKI in causing DDIs between opioids and benzodiazepines [13].

\subsection{Pharmacodynamic Interactions}

The combination of opioids and benzodiazepines has been defined as a risk factor for adverse effects. Concomitant opioid and benzodiazepine prescriptions have been proven to increase the risk of overdose [14, 15]. In 2015, benzodiazepines were reported to be involved in nearly $30 \%$ of opioid overdose deaths in the US, and thus use and misuse of benzodiazepines was considered to contribute significantly to the opioid overdose epidemic [16]. In a previous study, it was reported that $80 \%$ of the patients, in addition to opioids, also received benzodiazepines, and that the overdose rate was ten times higher among those that were co-prescribed benzodiazepines compared to those dispensed opioid analgesics alone [9]. Between 2002 and 2014 , the number of individuals with an opioid analgesic prescription and a co-prescription of benzodiazepines increased by $41 \%$ in US outpatients [17]. Caughey et al. reported that oxycodone was the second most common dispensed opioid, after codeine-paracetamol, holding $25 \%$ of all dispensed opioids in Australia. Nearly 45\% of the patients prescribed oxycodone had a concomitant prescription of diazepam [18]. Bech et al. studied the association between drug-induced cause of death and benzodiazepines among patients in Norway receiving opioid agonist treatment, and found that $76 \%$ of the cases had at least one benzodiazepine on board at time of death [19]. Combined use of alcohol and opioids is also associated with PDI effects, causing increased risk for respiratory depression. Vowles et al. studied the misuse of alcohol in patients prescribed opioids for chronic pain and found that $23 \%$ reported that they were misusing both opioids and alcohol, $38 \%$ opioid misuse, and $36 \%$ reported no misuse [20]. In a study regarding buprenorphine by Hakkinen et al., it was found that alcohol was significantly more common in fatal buprenorphine intoxications (58\%) than in other causes of death (32\%) suggesting a PDI [21].

Despite the large amount of work that has been put into the study of oxycodone in the clinical setting, the significance of possible PDI and PKI in postmortem cases involving oxycodone is limited. Thus, the main objective of this study was to investigate differences in prescription prevalence for and toxicological findings of PDI and PKI drugs in oxycodone-related deaths. We also aimed to investigate the differences in prevalence of oxycodone prescriptions, the detected postmortem oxycodone concentrations and oxycodone doses prescribed between fatal oxycodone-related intoxications and other causes of death.

\section{Methods}

\subsection{Data Sources}

In Sweden, a forensic autopsy is requested by the police if death is considered unnatural or unexplained. At the National Board of Forensic Medicine, the autopsy case work is conducted in one of six units for forensic medicine, and the toxicological analysis of femoral blood is conducted at a centralized national laboratory. Data from all Swedish forensic autopsies are stored in the National Board of Forensic Medicine database and includes demographics, autopsy findings, and toxicological findings. Information of medical history were retrieved from the Swedish National Patient Register holding information of in- and out-patient visits [22, 23]. Data of prescribed and dispensed drugs conducted at a Swedish pharmacy were drawn from the Swedish Prescribed Drug Register [24]. The Swedish National Patient Register and the Swedish Prescribed Drug Register are held by the National Board of Health and Welfare. The 12-digit personal identification number unique for each Swedish citizen and residents was used to link data between the databases [25].

\subsection{Study Population}

This retrospective nationwide study included forensic autopsy cases from 2012 through 2018, in which oxycodone [Anatomical Therapeutic Chemical (ATC) groups N02AA05, N02AA55, and N02AA56] was detected and quantified in postmortem femoral blood in individuals with a valid personal identification number $(n=1236)$. Cases were stratified into three groups based on the cause of death determined by the forensic pathologist (Fig. 1). The INTOX group included all fatal intoxication with oxycodone as the only cause of death or oxycodone contributing to death $(n=451)$. The NONINTOX group represented all other causes of death except intoxication $(n=630)$. The third group, OTHER, contained intoxications where oxycodone was considered an insignificant finding $(n=155)$. This group was not included in the data analysis.

\subsection{Definition of Variables}

A dispense of oxycodone from a valid prescription within 1 year before death was defined as an oxycodone prescription. The dose per day was defined by the total dose during 1 year before death from first to last purchase divided by number of days between the first and last purchases. Subjects with a single purchase within one year before death or with two purchases on the same day were not included in the analysis for oxycodone dose. Nutritional status was defined 
based on body mass index in $\mathrm{kg} / \mathrm{m}^{2}$ : underweight $(<18.5)$, normal weight (18.5-24.9), pre-obesity (25.0-29.9), and obesity ( $\geq 30.0$ ) [26]. The Swedish clinical guidelines developed for healthcare professional on DDIs were used to classify a substance as a PKI [26]. Substances were included as a PKI if (1) the combination with oxycodone was not recommended, or (2) the combination with oxycodone require adjustment of dose for oxycodone [27]. Prescription and dispense within 1 year before death were investigated for the following PKI substances that can act as a CYP3A4 inhibitor: bocaprevir, cyclosporine, dronedarone, erythromycin, idealisib, itraconazole, ketoconazole, clarithromycin, cobicistat, posaconazole, crizotinib, ritonavir, telaprevir, telithromycin, troleandomycin, and voriconazole. Drugs other than oxycodone belonging to the ATC groups N02A and N05 were considered PDI drugs. In N02A, we included ATC codes N07BC01 (buprenorphine), N07BC02 (methadone), and N07BC51 (buprenorphine). In N05, we included the ATC code N03AE01 (clonazepam). Alcohol (ethanol) was also defined as a PDI drug. Co-findings in femoral blood were defined as present when the parent drug and/or its metabolites were detected. Drugs that can be both a metabolite and a parent drug (e.g., morphine) were evaluated and allocated to the most probable parent drug (e.g., codeine). Visits to a hospital or other care unit were either a visit as an in- or out-patient care visit. Based on the diagnosis of a psychiatrist, the frequency of two diagnoses in the study population were explored: "Mood disorders" (ICD 10 codes F30-39) and "Mental and behavioral disorders due to psychoactive substance use" (ICD 10 codes F10-19) investigating two hypotheses; if substance abuse was correlated with fatal intoxication, and if mood affective disorders were correlated to suicide. These diagnoses are referred to as "Moodor behavioral diagnoses" throughout this paper.

\subsection{Statistical Analysis}

The statistics in this study are primarily descriptive. Categorical variables are presented by count and percentage, while continuous variables are presented by medians and percentiles $\left(P_{0} /\right.$ minimum, $P_{5}, P_{95}$ and $P_{100} /$ maximum). Bivariate analyses were used for group comparisons. For two group comparisons of continuous variables, the Mann-Whitney $U$ test was used for comparisons of distributions, while Mood's Median test was used for median comparisons. Simple logistic regressions were applied for two group comparisons of categorical variables. Resulting odds ratio (OR) are presented together with $95 \%$ confidence interval (CI). For categorical data with more than two groups, Chi-square tests were used. All statistical analyses were performed using IBM SPSS ${ }^{\circledR}$ Statistics v.27.0.0.0 (IBM, Armonk, NY, USA), and a $p$ value $<0.05$ (with Bonferroni correction when applicable) was considered as statistically significant.

\subsection{Ethical Approval}

The procedure to link the three databases, National Board of Forensic Medicine Database, Swedish Prescribed Drug Register, and Swedish National Patient Register, was performed at the National Board of Health and Welfare. During the same procedure, the personal identification number was replaced by a unique serial number to anonymize the cases in the study. The study was approved by the Regional Ethics Review Board in Linköping, Sweden, No. 2016/489-31 including extension 2018/577-32.

\section{Results}

In all, 1081 forensic autopsy cases were included in the analysis, representing $87 \%$ of all 1236 oxycodone findings during 2012-2018 (Table 1; Fig. 1). In 451 cases (42\%), the cause of death was intoxication related to oxycodone and in 630 cases $(58 \%)$, the cause of death was causes other than intoxication.

\subsection{Oxycodone Prescriptions and Blood Concentrations}

Of all 1081 cases, 34\% did not hold a valid prescription within 1 year before death (Table 2). The INTOX group was less likely to have oxycodone prescribed within 1 year before death ( $41 \%$ vs. $30 \%$, OR 0.62, CI 0.48-0.80) (Fig. 2). An oxycodone dose per day was calculated in 598 cases (55\%) based on prescribed dose. Of the 711 cases that had a prescription for oxycodone, 113 had only one or two dispenses within 1 year before death and were excluded from the analysis of prescribed oxycodone dose per day. The median oxycodone dose in the INTOX group, $33 \mathrm{mg} /$ day (range $0.20-1197 \mathrm{mg} /$ day), was significantly higher ( $p=0.017$ ) compared to the median in the NONINTOX group, $26 \mathrm{mg}$ /day (range 0.30-723 $\mathrm{mg} /$ day) (Table 2). The median oxycodone concentration in femoral blood, $0.30 \mu \mathrm{g} / \mathrm{g}$ (range $0.005-83 \mu \mathrm{g} / \mathrm{g}$ ) was significantly higher $(p<0.001)$ in the INTOX group compared to the median concentration in the NONINTOX group, 0.050 $\mu \mathrm{g} / \mathrm{g}$ (range $0.005-7.1 \mu \mathrm{g} / \mathrm{g}$ ) (Table 2). There was no significant difference in oxycodone concentration between cases that had a prescription (range $0.005-83 \mu \mathrm{g} / \mathrm{g}$ ) compared to those that did not have a prescription (range $0.005-17 \mu \mathrm{g} / \mathrm{g}$ ). The median concentration was $0.10 \mu \mathrm{g} / \mathrm{g}$ in both groups. The prevalence of cases in the age groups $<35,36-50,51-64$, and $\geq 65$ years without an oxycodone prescription is presented in Fig. 3. There were significant differences between age group and oxycodone prescription $(p<0.001)$. Among the 370 cases that did not have a prescription, $55 \%$ were $<50$ years old. Among the 711 cases that had an oxycodone prescription within 1 year before death, $70 \%$ were $>50$ years 
Table 1 Demographic data of the 1081 cases included in the study

\begin{tabular}{|c|c|c|c|}
\hline Variable & INTOX & NONINTOX & Total \\
\hline Cases & $451(42 \%)$ & $630(58 \%)$ & $1081(100 \%)$ \\
\hline \multicolumn{4}{|l|}{ Age group } \\
\hline$<18$ & $1(0.2 \%)$ & $2(0.3 \%)$ & $3(0.3 \%)$ \\
\hline $18-35$ & $115(26 \%)$ & $72(11 \%)$ & $187(17 \%)$ \\
\hline $36-50$ & $156(35 \%)$ & $75(12 \%)$ & $231(21 \%)$ \\
\hline $51-64$ & $105(23 \%)$ & $198(31 \%)$ & $303(28 \%)$ \\
\hline$\geq 65$ & $74(16 \%)$ & $283(45 \%)$ & $357(33 \%)$ \\
\hline \multicolumn{4}{|l|}{ Sex } \\
\hline Female & $173(38 \%)$ & $197(31 \%)$ & $370(34 \%)$ \\
\hline Male & $275(61 \%)$ & $433(69 \%)$ & $708(66 \%)$ \\
\hline Missing & $3(0.7 \%)$ & 0 & $3(0.3 \%)$ \\
\hline \multicolumn{4}{|l|}{ Body mass index } \\
\hline Underweight $(<18.5)$ & $16(3.5 \%)$ & $56(8.9 \%)$ & $72(6.7 \%)$ \\
\hline $\begin{array}{l}\text { Normal weight (18.5- } \\
24.9 \text { ) }\end{array}$ & $116(26 \%)$ & $236(38 \%)$ & $352(33 \%)$ \\
\hline Pre-obesity (25.0-29.9) & $151(34 \%)$ & $171(27 \%)$ & $322(30 \%)$ \\
\hline Obesity $(\geq 30.0)$ & $160(36 \%)$ & $149(24 \%)$ & $309(29 \%)$ \\
\hline Missing & $8(1.8 \%)$ & $18(2.9)$ & $26(2.4 \%)$ \\
\hline \multicolumn{4}{|l|}{ Place of death } \\
\hline Hospital/intensive care & $26(5.8 \%)$ & $136(22 \%)$ & $162(15 \%)$ \\
\hline Other care unit & $12(2.7 \%)$ & $27(4.3 \%)$ & $39(3.6 \%)$ \\
\hline Private home & $373(83 \%)$ & $377(60 \%)$ & $750(69 \%)$ \\
\hline Other/unknown & $35(7.8 \%)$ & $71(11 \%)$ & $106(9.8 \%)$ \\
\hline Missing & $5(1.1 \%)$ & $19(3 \%)$ & $24(2.2 \%)$ \\
\hline \multicolumn{4}{|l|}{ Manner of death } \\
\hline Natural (disease) & $1^{\mathrm{a}}(0.2 \%)$ & $346(55 \%)$ & $347(32 \%)$ \\
\hline Accident & $182(40 \%)$ & $116(18 \%)$ & $298(28 \%)$ \\
\hline Suicide & $138(31 \%)$ & $136(22 \%)$ & $274(25 \%)$ \\
\hline $\begin{array}{l}\text { Homicide/police interven- } \\
\text { tion }\end{array}$ & 0 & $5(0.8 \%)$ & $5(0.5 \%)$ \\
\hline Unclear & $130(29 \%)$ & $27(4.3 \%)$ & $157(15 \%)$ \\
\hline
\end{tabular}

Number of cases, age group (year), sex, body mass index $\left(\mathrm{kg} / \mathrm{m}^{2}\right)$, place of death, and manner of death are given for oxycodone-related deaths (INTOX), deaths from other causes than intoxication (NONINTOX), and for all cases

${ }^{a}$ Cause of death: drug dependence (ICD9 code 304)

old. Both immediate release oxycodone and controlled release oxycodone were prescribed to the 711 subjects who had an oxycodone prescription. Immediate release oxycodone alone was prescribed to 121 individuals (17\% of 711) and controlled release oxycodone alone was prescribed to 166 individuals (23\% of 711). Both immediate and controlled release oxycodone were prescribed to 424 individuals ( $60 \%$ of 711 ).

\subsection{Prescriptions and Findings of Pharmacokinetically Interacting Drugs}

One of the study's main questions was to investigate the prevalence of prescribed PKI drugs in the population. A prescription of a CYP3A4 inhibitor was only found in $1.3 \%$ of the whole population ( 15 cases), 7 cases $(2 \%)$ in the INTOX group, and 8 cases $(1 \%)$ in the NONINTOX group (Table 2; Fig. 2). The prescribed drugs were erythromycin (7 cases), cyclosporine (4 cases), clarithromycin, and ritonavir ( 2 cases each). No individual was prescribed more than one of the drugs within 1 year before death. The routine screening method for postmortem blood did not include these CYP3A4 inhibitors but included four substances that can act as a CYP3A4 inducer: phenytoin, carbamazepine, efavirenz, and primidone. Phenytoin was found in femoral blood in 2 cases in the NONINTOX group, and carbamazepine was found in 10 cases, 4 in the INTOX group, and 6 in the NONINTOX group.

\subsection{Prescription of Pharmacodynamically Interacting Drugs}

A PDI drug was prescribed to $79 \%$ of the 1081 cases. The INTOX group was more likely to have a PDI drug dispensed than the NONINTOX group ( $84 \%$ vs. $76 \%$, OR $1.7, \mathrm{CI}$ 1.2-2.3) (see Table 2; Fig. 2). Drugs in the ATC group N05 were more often prescribed than drugs in N02A (40\% vs. $6 \%$ ). Prescription of N05 and N02A medications in combination were found in $33 \%$ of the cases (Table 2). The most prevalent prescribed PDI drug was zopiclone, with $41 \%$ of the cases having at least one prescription within 1 year before death. Thereafter, came oxazepam $23 \%$, propiomazine $23 \%$, diazepam $21 \%$, and zolpidem $21 \%$. Within 1 year before death, multiple PDI drugs were prescribed. Up to 15 prescriptions were found in the INTOX group, and up to 14 different prescriptions in the NONINTOX group. An oxycodone prescription accompanied by a minimum of one PDI drug prescription within 1 year before death was found in $90 \%$ and $83 \%$ of the INTOX and NONINTOX groups, respectively. The most prevalent co-prescribed drugs were zopiclone, oxazepam, propiomazine, diazepam, and zolpidem. Cases in the INTOX group holding an oxycodone prescription were more likely to have a PDI drug prescribed compared to the cases in the NONINTOX group (OR 2.0, CI 1.2-3.1).

\subsection{Co-findings of Pharmacodynamically Interacting Drugs}

At the time of death, a PDI drug was found in femoral blood in $81 \%$ of all 1081 cases: $94 \%$ in the INTOX group and $72 \%$ in the NONINTOX group (Table 3; Fig. 2). Subjects in the INTOX group were more likely to have a PDI drug in their system (OR 5.6, CI 3.7-8.5). It was more likely that subjects in the INTOX group had a co-finding of one or more N05 drugs in femoral blood (OR 4.0, CI 3.0-5.3), alcohol (OR 2.1, CI 1.6-2.8), or N02A drug (OR 1.3, CI 1.0-1.7) 
Table 2 Number of oxycodone prescriptions within 1 year before death

\begin{tabular}{llll}
\hline Variable & INTOX $(n=451)$ & NONINTOX $(n=630)$ & Total $(n=1081)$ \\
\hline Oxycodone prescribed & $268(59 \%)$ & $443(70 \%)$ & $711(66 \%)$ \\
Oxycodone dose (mg/day) & $227(50 \%)$ & $371(59 \%)$ & $598^{\mathrm{a}}(55 \%)$ \\
Median (range) & $33(0.20-1197)$ & $26(0.30-723)$ & $30(0.20-1197)$ \\
5-95 percentile & $4.0-203$ & $3.0-155$ & $3.1-186$ \\
Oxycodone concentration ( $\mu \mathrm{g} / \mathrm{g})$ & & & \\
Median (range) & $0.30(0.005-83)$ & $0.050(0.005-7.1)$ & $0.10(0.005-83)$ \\
5-95 percentile & $0.020-2.1$ & $0.007-0.39$ & $0.008-1.0$ \\
Any PKI prescribed & $7(2 \%)$ & $8(1 \%)$ & $15(1 \%)$ \\
Any PDI prescribed & $379(84 \%)$ & $476(76 \%)$ & $855(79 \%)$ \\
Only N05 ${ }^{\mathrm{c}}$ prescribed & $184(41 \%)$ & $250(40 \%)$ & $434(40 \%)$ \\
Only N02A ${ }^{\mathrm{d}}$ prescribed & $17(4 \%)$ & $43(7 \%)$ & $60(6 \%)$ \\
Both N05+N02A prescribed & $178(40 \%)$ & $183(29 \%)$ & $361(33 \%)$ \\
\hline
\end{tabular}

Median, range, and 5-95\% percentile for calculated oxycodone dose per day and oxycodone concentrations found in femoral blood. Number of prescriptions of pharmacokinetically interacting and pharmacodynamically interacting drugs within 1 year before death. Data are divided by oxycodone-related deaths (INTOX), deaths from other causes than intoxication (NONINTOX), and for all cases

$P K I$ pharmacokinetically interacting, $P D I$ pharmacodynamically interacting

${ }^{a}$ For 113 cases, a daily dose could not be calculated (see Sect. 2.3 for definition of oxycodone dose).

${ }^{\mathrm{b}}$ The five most common PDI drugs were zopiclone, oxazepam, propiomazine, diazepam, and zolpidem.

${ }^{\mathrm{c}} \mathrm{N} 05$ includes also N03AE01 (clonazepam)

${ }^{\mathrm{d}} \mathrm{N} 02 \mathrm{~A}$ includes also N07BC01 (buprenorphine), N07BC02 (methadone), and N07BC51 (buprenorphine) compared to the NONINTOX group. In addition to alcohol and drugs available as medications, small numbers of nonpescription benzodiazepines and non-prescription opioids were also detected in the population. These were diclazepam $\left(n_{\text {NONINTOX }}=1\right)$, etizolam $\left(n_{\text {INTOX }}=4\right)$, phenazepam $\left(n_{\text {NONINTOX }}=1\right)$, flualprazolam $\left(n_{\text {INTOX }}=1, \mathrm{~N}_{\text {NONINTOX }}=\right.$ $1)$, flubromazolam $\left(n_{\text {INTOX }}=3, \mathrm{~N}_{\text {NONINTOX }}=1\right)$, norfludiazepam $\left(n_{\text {INTOX }}=6, \mathrm{~N}_{\text {NONINTOX }}=2\right)$, pyrazolam $\left(n_{\text {INTOX }}\right.$ $=2$ ), bromazepam (including the metabolite 3-OH-bromazepam) $\left(n_{\text {INTOX }}=1\right)$, 4-fluoroisobutyrfentanyl $\left(n_{\text {INTOX }}=\right.$ $1)$, acrylfentanyl $\left(n_{\text {INTOX }}=1\right)$, cyclopropylfentanyl $\left(n_{\text {INTOX }}\right.$ $\left.=3, \mathrm{~N}_{\text {NONINTOX }}=1\right)$, methoxyacetylfentanyl $\left(n_{\text {INTOX }}=1\right)$, MT-45 $\left(n_{\text {INTOX }}=1\right)$, and mitragynin $\left(n_{\text {INTOX }}=1\right)$. In supplemental Figure 1, co-findings of PDI drugs in the two cause of death groups are shown. A drug from the ATC group N05 was the most common co-finding in both the INTOX and NONINTOX groups, $36 \%$ and $31 \%$, respectively. Combinations of both N05 and N02A were found in 23\% (INTOX) and $14 \%$ (NONINTOX) of the cases. Zopiclone was the most common found PDI drug with 272 positive results, and the most prevalent N02A finding was morphine with 123 positive results. However, alcohol was present in femoral blood in 260 cases, making it the second most prevalent PDI drug co-finding.

\subsection{Diagnosis and National Patient Register}

Of the 1081 cases, $39 \%$ had visited an in- or out-patient care unit within 14 days before death, $32 \%$ in the INTOX group and $44 \%$ in the NONINTOX group. Of all cases, $89 \%$ had one or several diagnoses within 1 year before death. The occurrence of mental and behavioral disorders due to psychoactive substance use (F10-F19) and mood affective disorders (F30-F39) are shown in Table 4. We tested two hypotheses regarding diagnoses. Firstly, if substance use was correlated with fatal intoxication comparing the distribution of F10-F19 diagnoses between INTOX and NONINTOX groups. We found that subjects in the INTOX group were more likely to have a previous substance use diagnosis (29\% vs. $14 \%$, OR 2.6, CI 1.9-3.5). Secondly, we investigated if a mood affective disorder was correlated to suicide comparing the distribution of F30-F39 diagnosis between suicides and accidents. We found no significant difference with $14 \%$ of the suicides and $10 \%$ of the accidents presenting a mood affective diagnosis. We found no differences between those with a substance use disorder and those without a substance use diagnosis in the INTOX group regarding intake of multiple drugs or intake of sedative N05C.

\section{Discussion}

The main objective of this study was to investigate differences in prescriptions for and toxicological findings of PDI and PKI drugs in oxycodone-related deaths. The prescription of drugs that inhibit the primary route of metabolism of oxycodone via CYP3A4, increasing the risk for adverse effects, was very limited. Only $1 \%$ of the cases had been dispensed 


\section{INTOX}

a

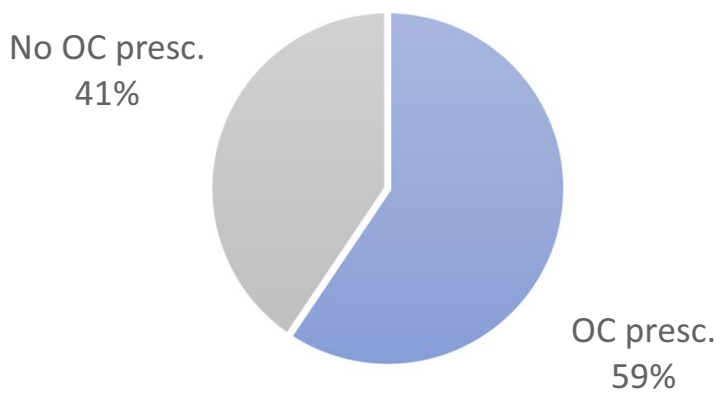

C

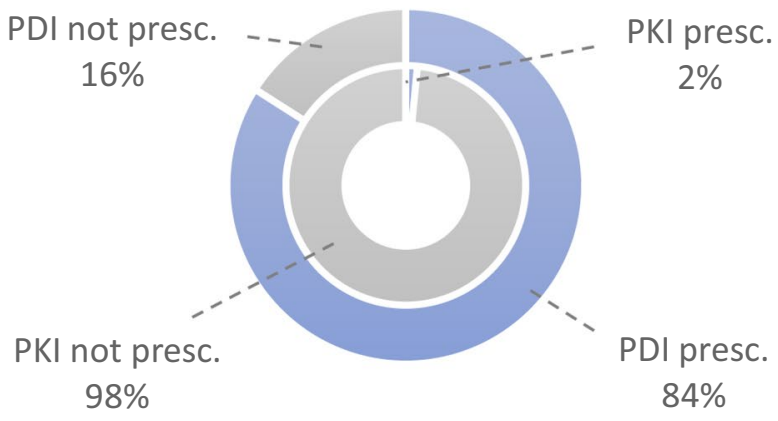

e

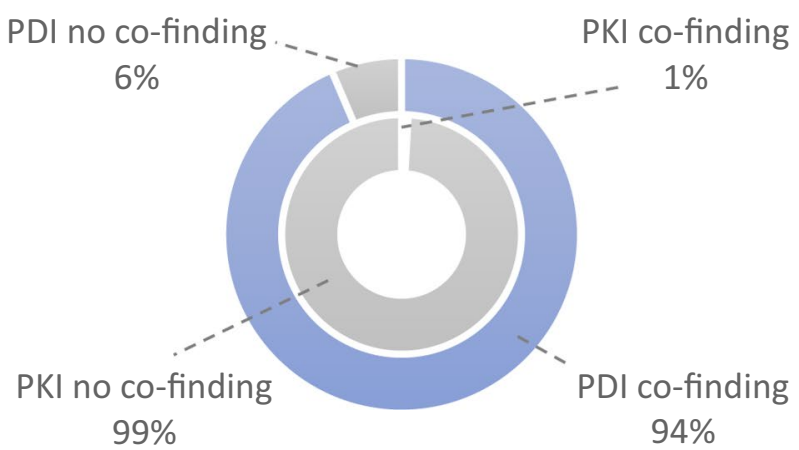

Fig. 2 a, b Cases with and without prescription of oxycodone $(O C)$ in oxycodone-related intoxications (INTOX) group and death by other causes (NONINTOX) group. The INTOX group was less likely to have oxycodone prescribed within 1 year before death (odds ratio 0.62 , CI 0.48-0.80). c, $\mathbf{d}$ Cases with and without prescription of pharmacodynamically interacting $(P D I)$ drug (outer ring) and pharmacokinetically interacting $(P K I)$ drug (inner ring) in the INTOX and NONINTOX groups. The INTOX group was more likely to have a

\section{NONINTOX}

b

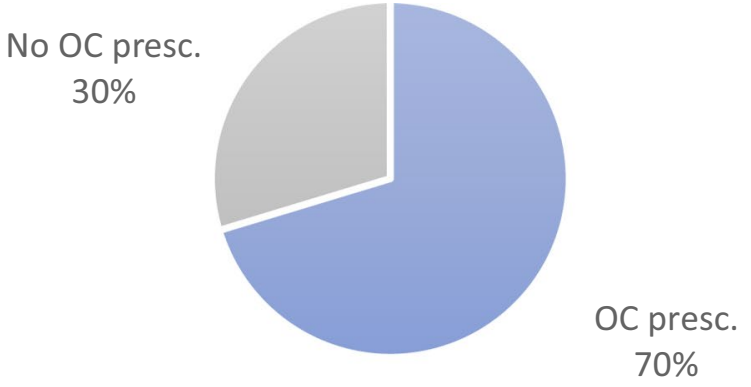

d

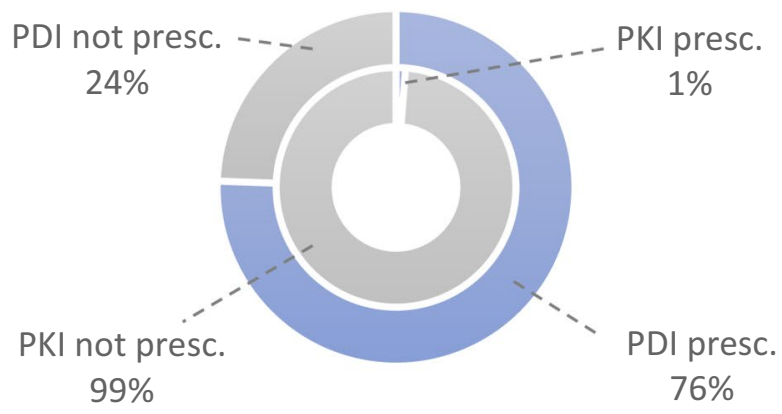

$f$

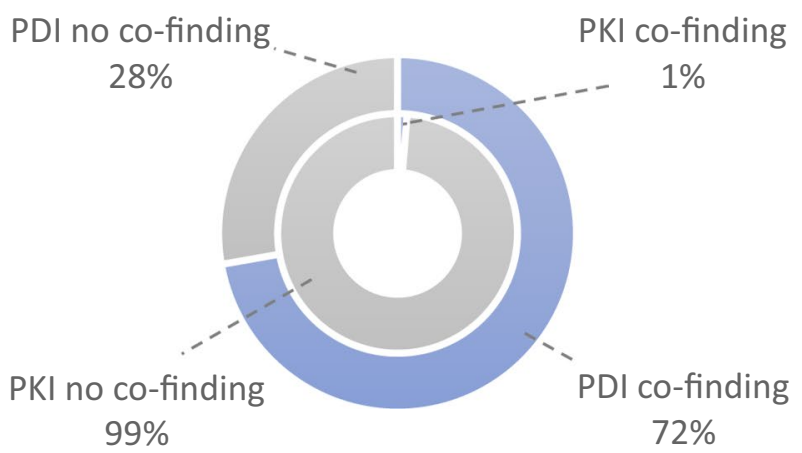

PDI drug prescribed (odds ratio 1.7, CI 1.2-2.3). The low number of prescribed PKI drugs ruled out statistical testing. e, f Cases with and without co-finding of PDI drug (outer ring) and PKI drug (inner ring) in the INTOX and NONINTOX groups. The INTOX group was more likely to have PDI drug on board at time of death (odds ratio 5.6, CI 3.7-8.5). The low number of PKI drugs found ruled out statistical testing 
Fig. 3 Distribution of cases (\%) with no prescription of oxycodone within 1 year before death in the age groups $<35(61 \%, n$ $=190), 36-50(39 \%, n=231)$, $51-64(25 \%, n=303)$, and $\geq 65$ $(25 \%, n=357)$ years

Table 3 Co-findings of pharmacodynamically interacting substances in femoral blood in cases related (INTOX) and unrelated (NONINTOX) to oxycodone, and in the whole study population

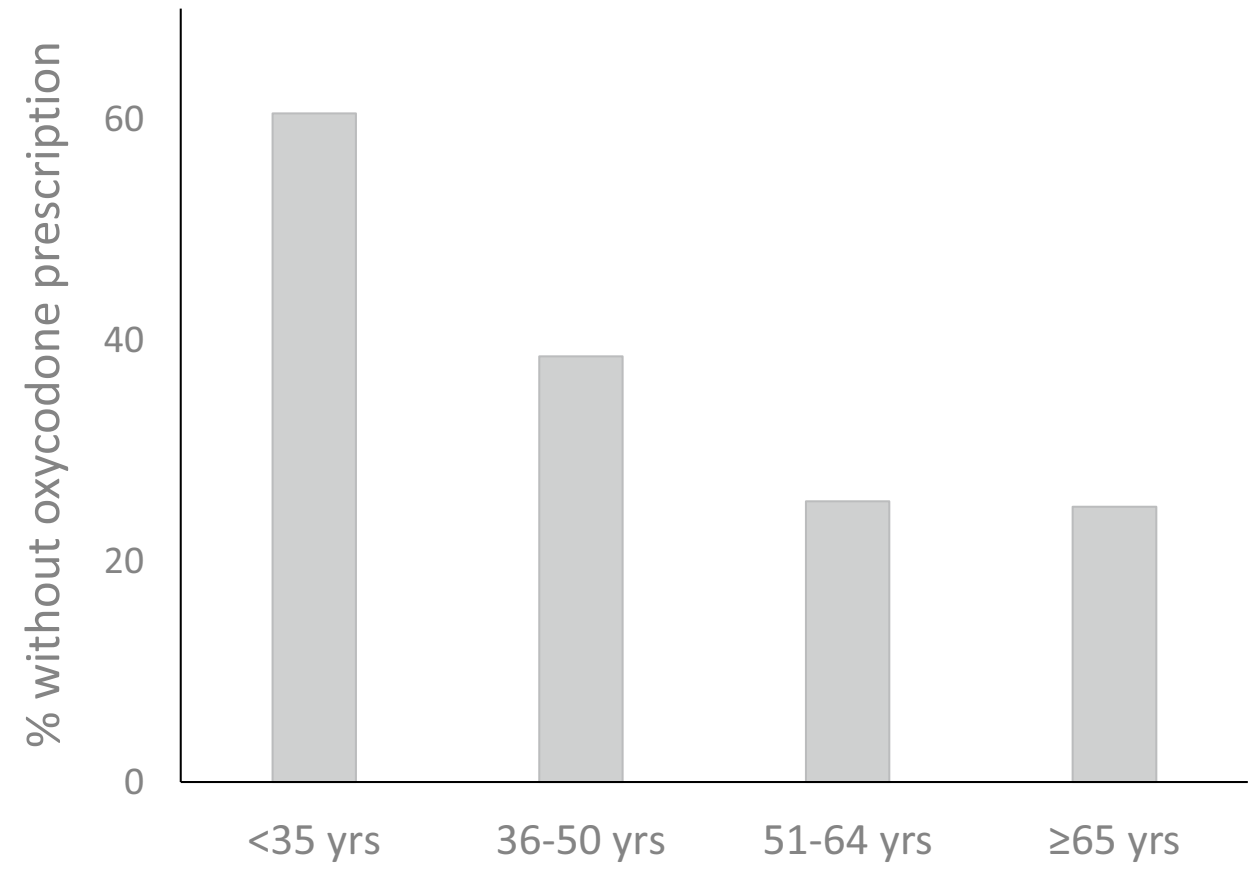

\begin{tabular}{llllll}
\hline & Total & INTOX $(n=451)$ & $\begin{array}{l}\text { NONINTOX } \\
(n=630)\end{array}$ & $p$ value & Odds ratio (95\% CI) \\
\hline PDI drug & $877(81 \%)$ & $422(94 \%)$ & $455(72 \%)$ & $<0.001$ & $5.6(3.7-8.5)$ \\
N05 drug & $718(66 \%)$ & $373(83 \%)$ & $345(55 \%)$ & $<0.001$ & $4.0(3.0-5.3)$ \\
N02A drug & $314(29 \%)$ & $147(33 \%)$ & $167(27 \%)$ & 0.03 & $1.3(1.0-1.7)$ \\
Alcohol & $260(24 \%)$ & $145(32 \%)$ & $115(18 \%)$ & $<0.001$ & $2.1(1.6-2.8)$ \\
\hline
\end{tabular}

Pharmacodynamically interacting drug indicates a finding of minimum one medication from ATC group N05, N02A, and/or alcohol

$P D I$ pharmacodynamically interacting, $C I$ confidence interval
Table 4 Number of cases with mood- or behavioral disorder in oxycodone-related deaths (INTOX) and deaths from other causes than intoxication (NONINTOX) as well as in different manners of death

\begin{tabular}{llll}
\hline Variable & F10-19 & F30-39 & F10-19 and F30-39 \\
\hline INTOX & $130(29 \%)$ & $63(14 \%)$ & $23(5 \%)$ \\
NONINTOX & $86(14 \%)$ & $47(8 \%)$ & $13(2 \%)$ \\
Natural (disease) & $50(14 \%)$ & $25(7 \%)$ & $9(3 \%)$ \\
Accident & $81(27 \%)$ & $29(10 \%)$ & $11(4 \%)$ \\
Suicide & $36(13 \%)$ & $37(14 \%)$ & $9(3 \%)$ \\
Homicide/police & 0 & 0 & 0 \\
$\quad$ intervention & & & $7(5 \%)$ \\
Unclear & $49(31 \%)$ & $19(12 \%)$ & \\
\hline
\end{tabular}

F10-F19 mental and behavioral disorders due to psychoactive substance use, F30-F39 mood affective disorders

a PKI drug in the year prior to death, indicating that this is a minor clinical problem. However, the small study population of 1086 cases should be taken into consideration. Our finding confirms the results from a recent larger study by Kahn et al. who also found very few co-prescriptions of PKI drugs in opioid overdoses [15]. Even though most of these antibacterial and immunosuppressive drugs are not in the primary panels of drugs to look for in a forensic autopsy, it will pose little problem for forensic toxicologists and medical examiners. Since the prevalence was so low, no comparison between the cause of death groups was performed.

Prescription of PDI drugs, on the other hand, was highly prevalent. In the total population, $79 \%$ had been dispensed one or more drugs enhancing the effects of oxycodone and increasing the risk of central nervous system (CNS) depressant effects including respiratory depression. It was a significant difference between the oxycodone-related fatal intoxications and other causes of death, where it was more frequent to have been dispensed a PDI drug in the fatal oxycodonerelated intoxications (OR 1.7). This is in accordance with a recent study by Bannon et al. where the authors found a prescription for the benzodiazepine alprazolam as the single 
best predictor for fatal opioid intoxication, whereas a prescription of an opioid alone was not correlated to opioid death [14]. In our study, zopiclone, oxazepam, propiomazine, diazepam, and zolpidem were the most prevalent PDI co-prescribed drugs. Although benzodiazepines are beneficial for patients with mental disorders, such as depression, anxiety, and post-traumatic stress disorder, co-prescription of opioids and benzodiazepines constitutes a highly risky practice. The pharmacodynamic interaction between benzodiazepines and opioids is related to the synergistic effects on CNS depression increasing the risk of respiratory depression [28].

A retrospective study by Griggs et al. aimed to characterize the prescribing of opioids and benzodiazepines to patients who had previously presented with an opioid or benzodiazepine overdose. They found that only a small part $(0.9 \%)$ of all prescriptions during 1 month were prescribed to patients with previous documented overdoses. Still, 404 patients with an overdose within 2 years were prescribed opioids and/or benzodiazepines [29]. In a study by Tori et al. in which the authors investigated the correlation between alcohol use and benzodiazepine prescription rates, on a population level, with the rate of involvement in opioid deaths, they found a positive correlation between benzodiazepine prescriptions and opioid deaths related to benzodiazepines. For prescription opioid-related deaths, there was an increase in co-involvement up to 2010 but then it flattened out at around $30 \%$ [30].

In summary, the risk of untoward effects from PKI drugs is low, suggesting well-managed protocols for treatment. On the other hand, despite the increased risk of adverse effects, co-prescription of PDI drugs was prevalent. This can be a result from so-called doctor shopping, when patients visit several prescribers to obtain multiple prescriptions. A way to avoid this is to implement national prescription registers from which physicians can retrieve information of a patient's prescriptions. If our results from the comparison of dispensed prescribed PDI drugs clearly pointed towards an increased risk of fatal intoxication, this became even more apparent when comparing the postmortem findings in femoral blood. At time of death, one or more PDI drugs were found in femoral blood in $81 \%$ of all cases, with a significant difference between the INTOX group (94\%) and the NONINTOX group (72\%). Similarly, Ogle et al. investigated benzodiazepine findings in oxycodone-related deaths $(n=154)$. The most common benzodiazepine was alprazolam which was significantly more prevalent among cases with oxycodone as a primary/contributory cause of death $(39.3 \%)$ compared to cases where oxycodone was incidental in the cause of death (15.8\%) [10]. In our study, cases in the INTOX were 5.6 times more likely to have a PDI substance present in femoral blood. Notable was that, in the INTOX group, there were more findings of drugs than there were prescriptions, but in the NONINTOX group, there were fewer findings than prescriptions. This may point towards misuse as a risk factor for severe adverse effects, including death. This is in accordance with Haukka et al., who observed that non-medical use of psychoactive prescription drugs was present in $71 \%$ of fatal intoxications. and that the most prevalent drugs were clonazepam, alprazolam, and tramadol [31].

We also aimed to investigate the prevalence of oxycodone prescriptions, and the postmortem oxycodone concentrations and oxycodone doses prescribed between fatal oxycodonerelated intoxications and other causes of death. It is known from previous publications that non-medical use of opioid prescription drugs is a widespread and alarmingly prevalent phenomenon that can lead to death $[3,9,31]$. In a study by Dasgupta et al., it was reported that only $50 \%$ of the opioidrelated overdose deaths had an opioid analgesic prescription active on the day of death [9]. Similarly, Pilgrim et al. found that $61 \%$ of oxycodone-related deaths lacked a prescription [3]. In our study, we found that $41 \%$ of the fatal intoxications did not have a valid prescription up to 1 year prior to death, a somewhat lower incidence than previous studies. However, we still found a significant difference between INTOX and NONINTOX, with the INTOX group less likely to have oxycodone prescribed. The higher incidence in the previous studies can be a result of missing data. For example, in the study by Pilgrim et al., prescriptions were not retrieved from a national database but from the information in the coroners' reports [3].

We also found a tendency that younger people were less likely to have an oxycodone prescription, with more than $60 \%$ of $<35$-year-olds lacking a prescription, whereas only $25 \%$ of people over 50 years old lacked one. A search in the literature for similar data was unsuccessful, indicating a knowledge gap. However, our findings indicate a high degree of non-medical use among younger people. A high incidence of diversion of medications pose a possible threat to public health and should be addressed by authorities.

In our study, doses between groups were significantly different, with a median of $33 \mathrm{mg} / \mathrm{day}$ among the fatal intoxications and $26 \mathrm{mg} /$ day in those where the cause of death was unrelated to oxycodone. However, there was a very wide range in prescribed doses in both groups, a finding that can be explained by the severity of pain as well as tolerance built up among those who are on long-term prescriptions. Our study design did not allow for an insight into how doses can be correlated to mortality, but, in a study by Dasgupta et al., they found a gradually increasing mortality rate with increased daily doses [9]. However, the distinct elevation in rate appeared above $200 \mathrm{mg}$ of morphine equivalents per day, which is in the upper part of oxycodone doses in our population (Table 2).

Postmortem blood drug concentrations are an important parameter used to evaluate if a death can be attributed to 
acute toxicity. Indeed, there are numerous compilations of drug concentrations that may help the forensic toxicologist and pathologist in their interpretation [32-36]. For drugs like opioids, that are known to cause tolerance, blood concentrations may be of less importance, but there are still published data from larger datasets referring to toxic concentrations of oxycodone [10,37-39]. In a study by Ogle et al., they investigated 155 oxycodone-related deaths, and concluded that the blood concentration of oxycodone was significantly higher $(0.48 \mathrm{mg} / \mathrm{L})$ when the cause of death was attributed to oxycodone than when it was not $(0.16 \mathrm{mg} / \mathrm{L})$ [10]. Baker et al. reported median values of $0.56 \mathrm{mg} / \mathrm{L}(n$ $=55)$ and $0.26 \mathrm{mg} / \mathrm{L}(n=135)$ in drug-related and nondrug-related cases, respectively. A problem with any such compilations is that the concentration itself has been used by the medical examiner to decide whether or not a case is an intoxication, true also for our study. Indeed, in our study, the median oxycodone concentrations in femoral blood differed between groups, with significantly higher concentrations in the INTOX group $(0.30 \mu \mathrm{g} / \mathrm{g})$ compared to the NONINTOX group $(0.050 \mu \mathrm{g} / \mathrm{g})$. Söderberg et al. found higher median concentrations in their study: $0.70 \mu \mathrm{g} / \mathrm{g}$ in intoxication cases $(n=19)$ and $0.10 \mu \mathrm{g} / \mathrm{g}$ in nonintoxication/reference cases ( $n$ $=4$ ), but the small number of subjects may have influenced their medians [40].

By comparing the incidence of the diagnosis behavioral disorder due to psychoactive substance abuse (F10-F19 diagnoses) between INTOX and NONINTOX, we wanted to investigate if substance abuse was correlated with fatal intoxications. We found that intoxication cases were 2.6 more likely to have an F10-19 diagnosis. This agrees with established knowledge that drug abusers are at risk of accidental intoxications. Indeed, in our population, the dominating manner of death in INTOX was accident, whereas in NONINTOX it was natural (disease), followed by suicide (Table 1). Mood affective disorders have been connected to suicide, but we failed to find a correlation between the F30-F39 diagnosis and suicide.

A sensitivity analysis was conducted to test the robustness of using the time period " 1 year before death" for oxycodone prescription. The results from the binary logistic regression analysis for "oxycodone prescription 1 year/365 days before death" (OR 0.62, CI 0.48-0.80) were compared to oxycodone 180 days before death, oxycodone 540 days before death, and oxycodone 730 days before death. The ORs for 180,540 , and 730 days before death were between 0.61 and 0.63 , indicating 1 year/365 days to be a robust time frame.

It cannot be ruled out that individuals who had a visit at an inpatient or outpatient care unit were given oxycodone at the visit without prescription. Therefore, a sensitivity test was conducted, in which individuals visiting an inpatient or outpatient care unit within 14 days before death were included to the group of individuals who had an oxycodone prescription within 1 year before death. The results from the binary logistic regression analysis revealed an OR of 0.46 (CI 0.34-0.62) compared to 0.62 (CI 0.48-0.80). This indicated that the outcome was not affected by the administration of non-prescribed oxycodone in hospital care.

\section{Conclusions}

We set out to investigate the significance of pharmacokinetic and pharmacodynamic interactions in oxycodone-related deaths. We can conclude that drugs impairing oxycodone metabolism were very rarely prescribed and dispensed both in intoxications as well as in nonintoxications. In contrast, pharmacodynamically interacting drugs were frequently prescribed and dispensed, and even more frequent was a co-finding in fatal oxycodone-related intoxications, suggesting that the associated risk needs to be addressed in clinical practice. Compared to other deaths, persons dying from oxycodone-related intoxication were 5.6 times more likely to have other CNS depressant drugs on board and 1.7 times more likely to have them prescribed and dispensed. We also found that, in oxycodone-related deaths, a significantly higher number of cases did not have a prescription for oxycodone, suggesting misuse or abuse as a risk factor. In addition, both doses and concentrations of oxycodone were significantly higher in the intoxications compared to other causes of death. The relevance of these findings is not clear, since at least the concentrations of oxycodone were part of the postmortem data used to determine the cause of death. However, our results clearly indicate that concomitant use of oxycodone and other CNS depressants increases the risk of accidental adverse effects including death.

Supplementary Information The online version contains supplementary material available at https://doi.org/10.1007/s13318-021-00750-9.

\section{Declarations}

Funding The study was funded by the National Board of Forensic Medicine.

Conflicts of interest The authors declare no conflict of interest.

Availability of data and material Data are available on request.

Code availability Not applicable

Author contributions AKJ and JA conceived a general design and applied for ethical approval. AKJ, GJ, HG, JK, and RK conceptualized the research questions and designed the study. SG and AKJ coordinated the retrieval of data from registers. SG, AKJ and HG supervised the statistical analyses and GJ and RK curated and analyzed the data and wrote the draft manuscript. All authors contributed to the manuscript and approved the final version. 
Ethics approval The study was approved by the Regional Ethics Review Board in Linköping, Sweden, No. 2016/489-31 including extension 2018/577-32.

\section{Consent to participate Not applicable.}

Consent for publication Not applicable.

Open Access This article is licensed under a Creative Commons Attribution-NonCommercial 4.0 International License, which permits any non-commercial use, sharing, adaptation, distribution and reproduction in any medium or format, as long as you give appropriate credit to the original author(s) and the source, provide a link to the Creative Commons licence, and indicate if changes were made. The images or other third party material in this article are included in the article's Creative Commons licence, unless indicated otherwise in a credit line to the material. If material is not included in the article's Creative Commons licence and your intended use is not permitted by statutory regulation or exceeds the permitted use, you will need to obtain permission directly from the copyright holder. To view a copy of this licence, visit http://creativecommons.org/licenses/by-nc/4.0/.

\section{References}

1. Hauser W, Schug S, Furlan AD. The opioid epidemic and national guidelines for opioid therapy for chronic noncancer pain: a perspective from different continents. Pain Rep. 2017;2(3):e599. https://doi.org/10.1097/PR9.0000000000000599.

2. Manchikanti L, Helm S 2nd, Fellows B, Janata JW, et al. Opioid epidemic in the United States. Pain Physician. 2012;15(3):ES9-38.

3. Pilgrim, J. L., Yafistham, S. P., Gaya, S., Saar, E., et al. An update on oxycodone: lessons for death investigators in Australia. Forensic Sci Med Pathol. 2015. 11;1):3-12. https://doi.org/10.1007/ s12024-014-9624-x.

4. Shipton EA, Shipton EE, Shipton AJ. A review of the opioid epidemic: what do we do about it? Pain Ther. 2018;7(1):23-36. https://doi.org/10.1007/s40122-018-0096-7.

5. van Amsterdam J, Pierce M, van den Brink W. Is Europe facing an emerging opioid crisis comparable to the U.S.? Ther Drug Monit. 2021;43(1):42-51. https://doi.org/10.1097/FTD.00000 00000000789.

6. Backryd E, Heilig M, Hoffmann M. Opioid prescription changes in Sweden 2000-2015. Lakartidningen. 2017. p. 114. https:// www.ncbi.nlm.nih.gov/pubmed/28485763

7. Muller AE, Clausen T, Sjogren P, Odsbu I, et al. Prescribed opioid analgesic use developments in three Nordic countries, 20062017. Scand J Pain. 2019;19(2):345-53. https://doi.org/10.1515/ sjpain-2018-0307.

8. Centers for Disease Control and Prevention (CDC). https://www. cdc.gov/drugoverdose/data/prescribing/overdose-death-maps. html. Accessed 13 July 2021.

9. Dasgupta N, Funk MJ, Proescholdbell S, Hirsch A, et al. Cohort study of the impact of high-dose opioid analgesics on overdose mortality. Pain Med. 2016;17(1):85-98. https://doi.org/10.1111/ pme. 12907.

10. Ogle A, Moore K, Barrett B, Young MS, et al. Clinical history and characteristics of persons with oxycodone-related deaths in Hillsborough County, Florida in 2009. Forensic Sci Int. 2012;223(13):47-52. https://doi.org/10.1016/j.forsciint.2012.07.016.

11. Kinnunen M, Piirainen P, Kokki H, Lammi P, et al. Updated clinical pharmacokinetics and pharmacodynamics of oxycodone.
Clin Pharmacokinet. 2019;58(6):705-25. https://doi.org/10.1007/ s40262-018-00731-3.

12. Kim JH, Kim JY, Lee N, Yee J, et al. The impact of drug interactions on adverse effects of oral oxycodone in male geriatric patients. J Clin Pharm Ther. 2020;45(5):976-82. https://doi.org/ 10.1111/jcpt.13113.

13. Ji B, Liu S, Xue Y, He X, et al. Prediction of drug-drug interactions between opioids and overdosed benzodiazepines using physiologically based pharmacokinetic (PBPK) modeling and simulation. Drugs R D. 2019;19(3):297-305. https://doi.org/10. 1007/s40268-019-00282-3.

14. Bannon MJ, Lapansie AR, Jaster AM, Saad MH, et al. Opioid deaths involving concurrent benzodiazepine use: assessing risk factors through the analysis of prescription drug monitoring data and postmortem toxicology. Drug Alcohol Depend. 2021;225:108854. https://doi.org/10.1016/j.drugalcdep.2021. 108854.

15. Khan NF, Bykov K, Glynn RJ, Barnett ML, et al. Coprescription of opioids with other medications and risk of opioid overdose. Clin Pharmacol Ther. 2021. https://doi.org/10.1002/cpt.2314.

16. Votaw VR, Geyer R, Rieselbach MM, McHugh RK. The epidemiology of benzodiazepine misuse: a systematic review. Drug Alcohol Depend. 2019;200:95-114. https://doi.org/10.1016/j. drugalcdep.2019.02.033.

17. Hwang CS, Kang EM, Kornegay CJ, Staffa JA, et al. Trends in the concomitant prescribing of opioids and benzodiazepines, 2002-2014. Am J Prev Med. 2016;51(2):151-60. https://doi. org/10.1016/j.amepre.2016.02.014.

18. Caughey GE, Gadzhanova S, Shakib S, Roughead EE. Concomitant prescribing of opioids and benzodiazepines in Australia, 2012-2017. Med J Aust. 2019;210(1):39-40. https://doi.org/ $10.5694 / \mathrm{mja} 2.12026$.

19. Bech AB, Clausen T, Waal H, Vindenes V, et al. Post-mortem toxicological analyses of blood samples from 107 patients receiving opioid agonist treatment: substances detected and pooled opioid and benzodiazepine concentrations. Addiction. 2020. https://doi.org/10.1111/add.15211.

20. Vowles KE, Witkiewitz K, Pielech M, Edwards KA, et al. Alcohol and opioid use in chronic pain: a cross-sectional examination of differences in functioning based on misuse status. J Pain. 2018;19(10):1181-8. https://doi.org/10.1016/j.jpain.2018.04. 013.

21. Hakkinen M, Launiainen T, Vuori E, Ojanpera I. Benzodiazepines and alcohol are associated with cases of fatal buprenorphine poisoning. Eur J Clin Pharmacol. 2012;68(3):301-9. https://doi.org/ 10.1007/s00228-011-1122-4.

22. Jakobsson GL, Sternegard E, Olen O, Myrelid P, et al. Validating inflammatory bowel disease (IBD) in the Swedish National Patient Register and the Swedish Quality Register for IBD (SWIBREG). Scand J Gastroenterol. 2017;52(2):216-21. https://doi.org/10. 1080/00365521.2016.1246605.

23. Ludvigsson JF, Andersson E, Ekbom A, Feychting M, et al. External review and validation of the Swedish national inpatient register. BMC Public Health. 2011;11:450. https://doi.org/10.1186/ 1471-2458-11-450.

24. Wettermark B, Hammar N, Fored CM, Leimanis A, et al. The new Swedish Prescribed Drug Register-opportunities for pharmacoepidemiological research and experience from the first six months. Pharmacoepidemiol Drug Saf. 2007;16(7):726-35. https://doi.org/10.1002/pds.1294.

25. Ludvigsson JF, Otterblad-Olausson P, Pettersson BU, Ekbom A. The Swedish personal identity number: possibilities and pitfalls in healthcare and medical research. Eur J Epidemiol. 2009;24(11):659-67. https://doi.org/10.1007/s10654-009-9350-y. 
26. World Health Organization (WHO). https://www.euro.who.int/ en/health-topics/disease-prevention/nutrition/a-healthy-lifestyle/ body-mass-index-bmi. Accessed 24 Sept 2021.

27. 2021.https://janusmed.sll.se/interaktioner/substance/oxikodon. Accessed 7 Jan 2021.

28. Jones JD, Mogali S, Comer SD. Polydrug abuse: a review of opioid and benzodiazepine combination use. Drug Alcohol Depend. 2012;125(1-2):8-18. https://doi.org/10.1016/j.drugalcdep.2012. 07.004 .

29. Griggs C, Wyatt S, Wally MK, Runyon M, et al. Prescribing of opioids and benzodiazepines among patients with history of overdose. J Addict Med. 2019;13(5):396-402. https://doi.org/10.1097/ ADM.0000000000000513.

30. Tori ME, Larochelle MR, Naimi TS. Alcohol or benzodiazepine co-involvement with opioid overdose deaths in the United States, 1999-2017. JAMA Netw Open. 2020;3(4):e202361. https://doi. org/10.1001/jamanetworkopen.2020.2361.

31. Haukka J, Kriikku P, Mariottini C, Partonen T, et al. Non-medical use of psychoactive prescription drugs is associated with fatal poisoning. Addiction. 2018;113(3):464-72. https://doi.org/10.1111/ add. 14014

32. Jonsson AK, Soderberg C, Espnes KA, Ahlner J, et al. Sedative and hypnotic drugs-fatal and non-fatal reference blood concentrations. Forensic Sci Int. 2014;236:138-45. https://doi.org/10. 1016/j.forsciint.2014.01.005.

33. Ketola RA, Kriikku P. Drug concentrations in post-mortem specimens. Drug Test Anal. 2019;11(9):1338-57. https://doi.org/10. 1002/dta.2662.
34. Launiainen T, Ojanpera I. Drug concentrations in post-mortem femoral blood compared with therapeutic concentrations in plasma. Drug Test Anal. 2014;6(4):308-16. https://doi.org/10. 1002/dta.1507.

35. Lefrancois E, Reymond N, Thomas A, Lardi C, et al. Summary statistics for drugs and alcohol concentration recovered in postmortem femoral blood in Western Switzerland. Forensic Sci Int. 2021;325:110883. https://doi.org/10.1016/j.forsciint.2021. 110883.

36. Soderberg C, Wernvik E, Tillmar A, Spigset O, et al. Antipsychotics-postmortem fatal and non-fatal reference concentrations. Forensic Sci Int. 2016;266:91-101. https://doi.org/10.1016/j.forsc iint.2016.05.002.

37. Baker DD, Jenkins AJ. A comparison of methadone, oxycodone, and hydrocodone related deaths in Northeast Ohio. J Anal Toxicol. 2008;32(2):165-71. https://doi.org/10.1093/jat/32.2.165.

38. Cone EJ, Fant RV, Rohay JM, Caplan YH, et al. Oxycodone involvement in drug abuse deaths. II. Evidence for toxic multiple drug-drug interactions. J Anal Toxicol. 2004;28(7):616-24. https://doi.org/10.1093/jat/28.7.616.

39. Jakobsson G, Green H, Kronstrand R. Oxycodone concentrations and metabolic ratios in femoral blood from fatal intoxications and other causes of death using LC-MS-MS. J Anal Toxicol. 2021;45(2):124-33. https://doi.org/10.1093/jat/bkaa051.

40. Soderberg C, Tillmar A, Johansson A, Wernvik E, et al. The importance of sample size with regard to the robustness of postmortem reference values. Forensic Sci Int. 2020;311:110292. https://doi.org/10.1016/j.forsciint.2020.110292. 